Commun. Korean Math. Soc. 26 (2011), No. 1, pp. 79-88

DOI 10.4134/CKMS.2011.26.1.079

\title{
ON RANK ONE PERTURBATIONS OF THE UNILATERAL SHIFT
}

\author{
Eungil Ko AND Ji Eun LeE
}

\begin{abstract}
In this paper we study some properties of rank one perturbations of the unilateral shift operators $T=S+u \otimes v$. In particular, we give some criteria for eigenvalues of $T$. Also we characterize some conditions for $T$ to be hyponormal.
\end{abstract}

\section{Introduction}

Let $\mathcal{H}$ be a separable complex Hilbert space and let $\mathcal{L}(\mathcal{H})$ denote the algebra of all bounded linear operators on $\mathcal{H}$. If $T \in \mathcal{L}(\mathcal{H})$, we write $\sigma(T)$, and $\sigma_{p}(T)$ for the spectrum, and the point spectrum of $T$, respectively.

An operator $T \in \mathcal{L}(\mathcal{H})$ is said to be quasinormal if $T$ and $T^{*} T$ commute. Also, if $T=U|T|$ is the polar decomposition of $T$, then $T$ is quasinormal if and only if $U$ and $|T|$ commute. An operator $T \in \mathcal{L}(\mathcal{H})$ is said to be hyponormal if $T^{*} T \geq T T^{*}$ where $T^{*}$ is the adjoint of $T$. It is known that the class of hyponormal operators is a larger class containing normal and quasinormal operators. A spectral operator is an operator with a countably additive resolution of the identity defined on the Borel sets of the plane (see [6]) and an operator $T \in \mathcal{L}(\mathcal{H})$ is hypercyclic if there is a vector $x \in \mathcal{H}$ with dense orbit $\left\{x, T x, T^{2} x, \ldots\right\}$ (see [10]).

If $u$ and $v$ are nonzero vectors in $\mathcal{H}$, we write $u \otimes v$ for the operator of the rank one defined by

$$
(u \otimes v) x=\langle x, v\rangle u, x \in \mathcal{H},
$$

where $\langle$,$\rangle denotes the inner product of the Hilbert space \mathcal{H}$.

Let $\left\{e_{n}\right\}_{n=0}^{\infty}$ denote an orthonormal basis for $\mathcal{H}$ which will remain fixed throughout this paper and let $S \in \mathcal{L}(\mathcal{H})$ be the unilateral shift of multiplicity one defined by $S e_{n}=e_{n+1}$ for $n=0,1, \ldots$ Throughout the paper we suppose

Received December 19, 2009; Revised May 25, 2010.

2010 Mathematics Subject Classification. Primary 47A11; Secondary 47A10.

Key words and phrases. rank one perturbation, the unilateral shift and hyponormal operator.

This work was supported by the Korea Research Foundation Grant funded by the Korean Government (MOEHRD) (NRF-2009-351-C00005). 
that $u$ and $v$ are nonzero vectors in $\mathcal{H}$ and their expansions with respect to the orthonormal basis $\left\{e_{n}\right\}_{n=0}^{\infty}$ are

$$
u=\sum_{n=0}^{\infty} a_{n} e_{n} \text { and } v=\sum_{n=0}^{\infty} b_{n} e_{n},
$$

where $a_{n}$ and $b_{n}$ are nonzero coefficients for all nonnegative integer $n$.

We say that an operator $T \in \mathcal{L}(\mathcal{H})$ is a rank one perturbation of an operator if there exist the vectors $u$ and $v$ (defined above) in the Hilbert space $\mathcal{H}$ such that $T=S+u \otimes v$. E. Ionascu has studied the several properties of rank one perturbations of diagonal operators (see [8]). It is natural to consider the rank one perturbations of subnormal operators. Also, it is unknown whether these operators have nontrivial invariant subspaces. As the special case of these operators, we study some properties of rank one perturbations of the unilateral shift $T=S+u \otimes v$. In particular, we give some criteria for eigenvalues of $T$. Also we characterize some conditions for $T$ to be hyponormal.

\section{Main results}

First, we give some criteria for eigenvalues of $T=S+u \otimes v$.

Theorem 2.1. Let $T=S+u \otimes v$. Then $0 \notin \sigma_{p}(T)$. Furthermore, a nonzero point $\mu \in \mathbb{C}$ is an eigenvalue of $T$ if and only if

(i) $\sum_{n=0}^{\infty} \sum_{j=0}^{n} \frac{a_{n-j} \bar{b}_{n}}{\mu^{j+1}}=1$ and

(ii) $\sum_{n=0}^{\infty}\left|\sum_{j=0}^{n} \frac{a_{n-j}}{\mu^{j+1}}\right|^{2}<\infty$.

Proof. If $T x=0$, then $(S+u \otimes v) x=S x+\langle x, v\rangle u=0$. Hence we obtain the following equation

$$
\sum_{n=0}^{\infty} x_{n} e_{n+1}+\langle x, v\rangle \sum_{n=0}^{\infty} a_{n} e_{n}=0
$$

where $x=\sum_{n=0}^{\infty} x_{n} e_{n}$. If we solve this equation, then we get $\langle x, v\rangle=0$. Therefore $\sum_{n=0}^{\infty} x_{n} e_{n+1}=0$. Thus $x=0$. Hence $0 \notin \sigma_{p}(T)$.

Assume that $\mu \neq 0$. If $\mu \in \sigma_{p}(T)$, then there exists a nonzero vector $y$ such that $T y=\mu y$. Thus

$$
\langle y, v\rangle u=(\mu-S) y
$$

If $\langle y, v\rangle=0$, then $S y=\mu y$. It is a contradiction since $\sigma_{p}(S)=\emptyset$. Thus $\langle y, v\rangle \neq 0$. Set $x=-\frac{1}{\langle y, v\rangle} y$. Then $x \neq 0$ and

$$
(S-\mu) x=-\frac{1}{\langle y, v\rangle}(S-\mu) y=u .
$$

Hence $u \in \operatorname{ran}(S-\mu)$ and $\langle x, v\rangle+1=0$. Thus

$$
0=\langle x, v\rangle+1=\left\langle\sum_{n=0}^{\infty} x_{n} e_{n}, \sum_{m=0}^{\infty} b_{m} e_{m}\right\rangle+1=\sum_{n=0}^{\infty} x_{n} \bar{b}_{n}+1 .
$$


Since $u=(S-\mu) x$, the equation $\sum_{n=0}^{\infty} a_{n} e_{n}=(S-\mu) \sum_{n=0}^{\infty} x_{n} e_{n}$ holds. Hence

$$
\left\{\begin{array}{l}
a_{0}=-\mu x_{0} \\
a_{n}=x_{n-1}-\mu x_{n}, \quad n=1,2, \ldots
\end{array}\right.
$$

If we solve this system with respect to $x_{n}$, then we obtain the following equations:

$$
x_{n}=-\sum_{j=0}^{n} \frac{a_{n-j}}{\mu^{j+1}}, \quad n=0,1,2, \ldots .
$$

If we substitute (2) into (1), we get that

$$
1=\sum_{n=0}^{\infty} \sum_{j=0}^{n} \frac{a_{n-j} \bar{b}_{n}}{\mu^{j+1}}
$$

In order to get (ii), from $\|x\|<\infty$ and (2) we have

$$
\infty>\|x\|^{2}=\sum_{n=0}^{\infty}\left|x_{n}\right|^{2}=\sum_{n=0}^{\infty}\left|\sum_{j=0}^{n} \frac{a_{n-j}}{\mu^{j+1}}\right|^{2} .
$$

Conversely, if (i) and (ii) hold, set

$$
x=-\sum_{n=0}^{\infty}\left(\sum_{j=0}^{n} \frac{a_{n-j}}{\mu^{j+1}}\right) e_{n} .
$$

Then we obtain the following equations

$$
\begin{aligned}
\langle x, v\rangle+1 & =\left\langle-\sum_{n=0}^{\infty}\left(\sum_{j=0}^{n} \frac{a_{n-j}}{\mu^{j+1}}\right) e_{n}, \sum_{m=0}^{\infty} b_{m} e_{m}\right\rangle+1 \\
& =-\sum_{n=0}^{\infty}\left(\sum_{j=0}^{n} \frac{a_{n-j}}{\mu^{j+1}}\right) \bar{b}_{n}+1=0 .
\end{aligned}
$$

Hence $x \neq 0$ and

$$
(S-\mu) x=(S-\mu)\left(-\sum_{n=0}^{\infty}\left(\sum_{j=0}^{n} \frac{a_{n-j}}{\mu^{j+1}}\right) e_{n}\right)=u .
$$

Therefore

$$
T x=S x+\langle x, v\rangle u=\mu x .
$$

Thus $\mu \in \sigma_{p}(T)$.

Corollary 2.2. Let $T=S+u \otimes v$. If $\mu \in \sigma(T)$ and $|\mu|>1$, then $\mu \in \sigma_{p}(T)$. Hence (i) and (ii) in Theorem 2.1 hold. 
Proof. Assume that $\mu \in \sigma(T)$ and $|\mu|>1$. Since $\sigma(S)=\overline{\mathbb{D}}$ where $\mathbb{D}$ is the unit disc, it follows that $\mu \notin \sigma(S)$. Hence $S-\mu$ is invertible. Since $T-\mu=$ $S-\mu+u \otimes v=(S-\mu)\left[1+(S-\mu)^{-1} u \otimes v\right],(S-\mu)^{-1} u \otimes v$ is a rank one operator, and $T-\mu$ is not invertible, it follows that $-1 \in \sigma_{p}\left((S-\mu)^{-1} u \otimes v\right)$. Thus there exists a nonzero eigenvector $x$ such that $\left((S-\mu)^{-1} u \otimes v\right) x=-x$. So we get that $(u \otimes v) x=-(S-\mu) x$. Thus $(T-\mu) x=(S-\mu) x+(u \otimes v) x=0$. Hence $\mu \in \sigma_{p}(T)$. Thus (i) and (ii) in Theorem 2.1 hold.

Corollary 2.3. Let $T=S+u \otimes v$. If

$$
F(\mu)=\sum_{n=0}^{\infty} \sum_{j=0}^{n} \frac{a_{n-j} \bar{b}_{n}}{\mu^{j+1}}
$$

for all nonzero $\mu \in \sigma_{p}(T)$, then $F(\mu)$ converges absolutely.

Proof. Since

$$
\sum_{n=0}^{\infty}\left|\sum_{j=0}^{n} \frac{a_{n-j} \bar{b}_{n}}{\mu^{j+1}}\right| \leq\left(\sum_{n=0}^{\infty}\left|\sum_{j=0}^{n} \frac{a_{n-j}}{\mu^{j+1}}\right|^{2}\right)^{\frac{1}{2}}\left(\sum_{n=0}^{\infty}\left|b_{n}\right|^{2}\right)^{\frac{1}{2}}<\infty
$$

for all nonzero $\mu \in \sigma_{p}(T)$ by Theorem 2.1, $F(\mu)$ converges absolutely.

As an application of Theorem 2.1, we obtain the following example.

Example 2.4. Let

$$
T=S+\left(\sum_{n=0}^{\infty} \frac{1}{2^{n}} e_{n}\right) \otimes\left(\sum_{n=0}^{\infty} \frac{1}{n+1} e_{n}\right) .
$$

Then $\{2\} \subset \sigma_{p}(T)$.

Proof. We want to show that (i) and (ii) in Theorem 2.1 hold.

$$
\sum_{n=0}^{\infty} \sum_{j=0}^{n} \frac{\frac{1}{2^{n-j}} \frac{1}{n+1}}{2^{j+1}}=\sum_{n=0}^{\infty} \sum_{j=0}^{n} \frac{1}{(n+1) 2^{n+1}}=1 .
$$

Moreover,

$$
\sum_{n=0}^{\infty}\left|\sum_{j=0}^{n} \frac{\frac{1}{2^{n-j}}}{2^{j+1}}\right|^{2}=\sum_{n=0}^{\infty}\left(\frac{n+1}{2^{n+1}}\right)^{2}<\infty .
$$

Hence $2 \in \sigma_{p}(T)$ by Theorem 2.1 .

Next, we show that a rank one perturbation of the unilateral shift $S$ is not quasinormal. In [7, Problem 184] Halmos initiated that if $U$ is the unilateral shift, does there exist a compact operator $C$ such that $U+C$ is normal? He has proved that there is no rank one operator $u \otimes v$ such that $T=S+u \otimes v$ is normal where $S$ is the unilateral shift. Next we will consider the case of quasinormality of $T$. 
Proposition 2.5. Let $T=S+u \otimes v$. Then $T$ is not quasinormal.

Proof. If $T=S+u \otimes v$ is quasinormal, then

$$
\begin{aligned}
S^{*} S & =T^{*} T-\left(T^{*} u \otimes v\right)-\left(v \otimes T^{*} u\right)+\|u\|^{2}(v \otimes v) \\
& =T^{*} T-D
\end{aligned}
$$

where $D=\left(T^{*} u \otimes v\right)+\left(v \otimes T^{*} u\right)-\|u\|^{2}(v \otimes v)$. Then $D$ is an operator of finite rank. Since $T^{*} T-I=D$, it ensures that $\sigma\left(T^{*} T\right) \backslash\{1\} \subset \sigma_{p}\left(T^{*} T\right)$ by [7]. Since a Hermitian operator on a separable Hilbert space can have only countably many eigenvalues, it holds that $\sigma_{p}\left(T^{*} T\right)$ is countable. Since $\sigma(S)$ is the closed unit disc and $\sigma_{p}(S)=\emptyset, \sigma(T)$ can differ from the closed unit disc by the set of eigenvalues of $T$ only (i.e., $\sigma(T) \backslash \overline{\mathbb{D}}=\sigma_{p}(T)$ ) by [7]. It is known from [1] that if $T$ is quasinormal, then $T$ is unitarily equivalent to $N \oplus(P \otimes S)$ where $P$ is positive and $N$ is normal. Then $\sigma_{p}(T)=\sigma_{p}(N) \cup \sigma_{p}(P \otimes S)$. Since $\sigma_{p}(P \otimes S)=\sigma_{p}(P) \sigma_{p}(S)$ by $[2]$ and $\sigma_{p}(S)=\emptyset$, it follows that $\sigma_{p}(P \otimes S)=\emptyset$. Thus $\sigma_{p}(T)=\sigma_{p}(N)$. Since a normal operator on a separable Hilbert space can have only countably many eigenvalues, $\sigma_{p}(N)$ is countable. Hence $\sigma(T) \backslash \overline{\mathbb{D}}$ is countable and $\sigma\left(T^{*} T\right) \backslash[0,1]$ is countable. Thus $\sigma\left(T^{*} T\right)=[0,1]$ module countable sets. It contradicts to the countability of $\sigma\left(T^{*} T\right)$.

Corollary 2.6. If $T=S+u \otimes v$, then (i) neither $T^{*}$ nor $T$ is spectral, and (ii) $T$ is not hypercyclic.

Proof. (i) Since $\sigma_{p}\left(T^{*}\right)$ contains $\mathbb{D}$ by [14], it is uncountable. Hence by [6], $T^{*}$ is not spectral. Assume that $T=S+u \otimes v$ is spectral. Then $T^{*} T-I=F$ where $F=v \otimes T^{*} u+S^{*} u \otimes v$ is an operator of finite rank. With the same argument as in the proof of Theorem 2.5, we get a contradiction.

(ii) If $T$ is hypercyclic, then $\sigma_{p}\left(T^{*}\right)=\emptyset$ by [10]. But since $\sigma_{p}\left(T^{*}\right)$ contains $\mathbb{D}$ by [14], we have a contradiction.

An arbitrary operator $T \in \mathcal{L}(\mathcal{H})$ has a unique polar decomposition $T=$ $U|T|$, where $|T|=\left(T^{*} T\right)^{\frac{1}{2}}$ and $U$ is the appropriate partial isometry. Associated with $T$ there is a related operator $\tilde{T}=|T|^{\frac{1}{2}} U|T|^{\frac{1}{2}}$, called the Aluthge transform of $T$ (see [9]).

Corollary 2.7. If $T=S+u \otimes v$, then $T \neq \tilde{T}$.

Proof. If $T=\tilde{T}$, then $T$ must be quasinormal by [9]. So we have a contradiction.

Next we give a sufficient and necessary condition for rank one perturbations of the unilateral shift to be hyponormal.

Theorem 2.8. (i) Let $u$ and $v$ be linearly dependent. Then $T=S+u \otimes v$ is hyponormal if and only if

$$
\operatorname{Re}(\langle x, v\rangle\langle w, x\rangle) \geq-\frac{1}{2}\left|\left\langle x, e_{0}\right\rangle\right|^{2}
$$


holds where $w=\alpha S^{*} v-\bar{\alpha} S v$ and $\alpha=\frac{\langle u, v\rangle}{\|v\|^{2}}$.

(ii) Let $u$ and $v$ be linearly independent. Then $T=S+u \otimes v$ is hyponormal if and only if for all $x=y+z$ with $y \in \vee\{u, v\}$ and $z \in(\vee\{u, v\})^{\perp}$

$$
\operatorname{Re}\langle t, x\rangle+\frac{1}{2}\left(\|u\|^{2}|\langle y, v\rangle|^{2}-\|v\|^{2}|\langle y, u\rangle|^{2}\right) \geq-\frac{1}{2}\left|\left\langle x, e_{0}\right\rangle\right|^{2}
$$

holds where $t=\left\langle x, S^{*} u\right\rangle v-\langle x, u\rangle S v$.

Proof. (i) If $u$ and $v$ are linearly dependent, then there exists $\alpha \in \mathbb{C}$ such that $u=\alpha v$. Since $\alpha=\frac{\langle u, v\rangle}{\|v\|^{2}}$,

$$
\|v\|^{2} u \otimes u=|\alpha|^{2}\|v\|^{2} v \otimes v=\|u\|^{2} v \otimes v .
$$

Set $w=\alpha S^{*} v-\bar{\alpha} S v$. Then

$$
\begin{aligned}
\left\langle\left(T^{*} T-T T^{*}\right) x, x\right\rangle & =\left\langle\left(S^{*} S-S S^{*}\right) x, x\right\rangle+\langle(w \otimes v) x, x\rangle+\langle(v \otimes w) x, x\rangle \\
& =\left\langle\left(S^{*} S-S S^{*}\right) x, x\right\rangle+2 \operatorname{Re}\langle(w \otimes v) x, x\rangle \\
& =\left\langle\left(S^{*} S-S S^{*}\right) x, x\right\rangle+2 \operatorname{Re}(\langle x, v\rangle\langle w, x\rangle)
\end{aligned}
$$

for all $x \in \mathcal{H}$. Hence $T$ is hyponormal if and only if

$$
\operatorname{Re}(\langle x, v\rangle\langle w, x\rangle) \geq-\frac{1}{2}\left\langle\left(S^{*} S-S S^{*}\right) x, x\right\rangle=-\frac{1}{2}\left|\left\langle x, e_{0}\right\rangle\right|^{2} .
$$

(ii) Suppose that $u$ and $v$ are linearly independent. Set $t=\left\langle x, S^{*} u\right\rangle v-$ $\langle x, u\rangle S v$. Then $T=S+u \otimes v$ is hyponormal if and only if for any $x \in \mathcal{H}$

$$
\begin{aligned}
& \left\langle\left(T^{*} T-T T^{*}\right) x, x\right\rangle \\
= & \left\langle\left[\left(S^{*} S-S S^{*}\right) x+\left(S^{*} u \otimes v\right) x+\left(v \otimes S^{*} u\right) x+\left(\|u\|^{2} v \otimes v\right) x\right.\right. \\
& \left.\left.-(S v \otimes u) x-(u \otimes S v) x-\left(\|v\|^{2} u \otimes u\right) x\right], x\right\rangle \\
= & \left\langle\left(S^{*} S-S S^{*}\right) x, x\right\rangle+\left\langle x, S^{*} u\right\rangle\langle v, x\rangle-\langle x, u\rangle\langle S v, x\rangle \\
& +\langle x, v\rangle\left\langle S^{*} u, x\right\rangle-\langle u, x\rangle\langle x, S v\rangle+\|u\|^{2}|\langle x, v\rangle|^{2}-\|v\|^{2}|\langle x, u\rangle|^{2} \\
= & \left\langle\left(S^{*} S-S S^{*}\right) x, x\right\rangle+\left\langle\left\langle x, S^{*} u\right\rangle v-\langle x, u\rangle S v, x\right\rangle \\
& +\left\langle x, \overline{\left\langle S^{*} u, x\right\rangle} v-\overline{\langle u, x\rangle} S v\right\rangle+\|u\|^{2}|\langle x, v\rangle|^{2}-\|v\|^{2}|\langle x, u\rangle|^{2} \\
= & \left\langle\left(S^{*} S-S S^{*}\right) x, x\right\rangle+2 R e\langle t, x\rangle+\left(\|u\|^{2}|\langle x, v\rangle|^{2}-\|v\|^{2}|\langle x, u\rangle|^{2}\right) \\
= & \left|\left\langle x, e_{0}\right\rangle\right|^{2}+2 \operatorname{Re}\langle t, x\rangle+\left(\|u\|^{2}|\langle x, v\rangle|^{2}-\|v\|^{2}|\langle x, u\rangle|^{2}\right) \geq 0 .
\end{aligned}
$$

Let $\mathcal{H}=\vee\{u, v\} \oplus(\vee\{u, v\})^{\perp}$ where $\vee\{u, v\}:=\operatorname{span}\{u, v\}$. If $x=y+z$ with some $y \in \vee\{u, v\}$ and $z \in(\vee\{u, v\})^{\perp}$, then it clear that $\langle u, z\rangle=\langle v, z\rangle=0$. Hence from (3) we get that $T=S+u \otimes v$ is hyponormal if and only if

$$
\left|\left\langle x, e_{0}\right\rangle\right|^{2}+2 \operatorname{Re}\langle t, x\rangle+\left(\|u\|^{2}|\langle y, v\rangle|^{2}-\|v\|^{2}|\langle y, u\rangle|^{2}\right) \geq 0 .
$$

So we complete our proof.

Corollary 2.9. Let $u$ and $v$ be linearly independent with $\|u\|=\|v\|=1$ and let $x=c u+d v+z$ for some constant $c, d$ with $|d| \geq|c|$, and $z \in(\vee\{u, v\})^{\perp}$. 
If $\left\langle x, S^{*} u\right\rangle v=\langle x, u\rangle S v$ or $x=\left\langle x, S^{*} u\right\rangle v-\langle x, u\rangle S v$, then $T=S+u \otimes v$ is hyponormal.

Proof. Suppose that $u$ and $v$ are linearly independent with $\|u\|=\|v\|=1$. Let $x=c u+d v+z$ be with $|d| \geq|c|$ and $z \in(\vee\{u, v\})^{\perp}$. Then $c=\langle y, u\rangle$ and $d=\langle y, v\rangle$ where $y=c u+d v$. Since $|d| \geq|c|$, it clear that $|\langle y, v\rangle|^{2} \geq|\langle y, u\rangle|^{2}$. Set $t=\left\langle x, S^{*} u\right\rangle v-\langle x, u\rangle S v$. If $\left\langle x, S^{*} u\right\rangle v=\langle x, u\rangle S v$ or $x=\left\langle x, S^{*} u\right\rangle v-\langle x, u\rangle S v$, then $t=x$ or $t=0$. Hence

$$
\operatorname{Re}\langle t, x\rangle+\frac{1}{2}\left(|\langle y, v\rangle|^{2}-|\langle y, u\rangle|^{2}\right)+\frac{1}{2}\left|\left\langle x, e_{0}\right\rangle\right|^{2} \geq 0 .
$$

Therefore, $T$ is hyponormal from Theorem 2.8 .

Corollary 2.10. Let $T=S+u \otimes v$ be where $u$ and $v$ are linearly dependent. If $\gamma v=\alpha S^{*} v-\bar{\alpha} S v$ with $\alpha=\frac{\langle u, v\rangle}{\|v\|^{2}}$, then $\gamma=i \frac{2}{\|v\|^{4}} \operatorname{Im}\{\langle u, v\rangle\langle v, S v\rangle\}$ and $T$ is hyponormal.

Proof. If $\gamma v=\alpha S^{*} v-\bar{\alpha} S v$ with $\alpha=\frac{\langle u, v\rangle}{\|v\|^{2}}$, then $\gamma\|v\|^{2}=\alpha\left\langle S^{*} v, v\right\rangle-\bar{\alpha}\langle S v, v\rangle$. It follows that

$$
\begin{aligned}
\gamma\|v\|^{4} & =\langle u, v\rangle\left\langle S^{*} v, v\right\rangle-\overline{\langle u, v\rangle\langle v, S v\rangle} \\
& =2 i \operatorname{Im}\{\langle u, v\rangle\langle v, S v\rangle\} .
\end{aligned}
$$

So we have $\gamma=\frac{2 i}{\|v\|^{4}} \operatorname{Im}\{\langle u, v\rangle\langle v, S v\rangle\}$. Since $u$ and $v$ are linearly dependent, it suffices to show that $\operatorname{Re}\{\langle x, v\rangle\langle\gamma v, x\rangle\} \geq 0$. In fact, $\langle x, v\rangle\langle\gamma v, x\rangle=\gamma|\langle x, v\rangle|^{2}$. Hence $\operatorname{Re}\{\langle x, v\rangle\langle\gamma v, x\rangle\}=0$.

Example 2.11. Let $u=\sum_{n=0}^{\infty} \frac{1}{n+1} e_{n}$ and $v=\sum_{n=0}^{\infty} \frac{2 i}{n+1} e_{n}$. Then

$$
\gamma=i \frac{2}{\|v\|^{4}} \operatorname{Im}\{\langle u, v\rangle\langle v, S v\rangle\}=-i \frac{6}{\pi^{2}}
$$

and $S+\left(\sum_{n=0}^{\infty} \frac{1}{n+1} e_{n}\right) \otimes\left(\sum_{n=0}^{\infty} \frac{2 i}{n+1} e_{n}\right)$ is hyponormal.

Next we now turn to some properties of some operators in $\{T=S+u \otimes v\}^{\prime}$ where $\{T\}^{\prime}=\{A \in \mathcal{L}(\mathcal{H}) \mid T A=A T\}$. We start with the following theorem.

Proposition 2.12. Let $T=S+u \otimes v$. If $A \in\{T\}^{\prime}$ is a diagonal normal operator such that $A e_{k}=\gamma_{k} e_{k}$ for all $k \geq 0$, then either $A=\gamma I$ for some constant $\gamma$ or there are at most finitely many $j$ satisfying $a_{j+1} \bar{b}_{j}=-1$.

Proof. Since $A$ and $T$ commute, i.e., $A T=T A$, we obtain that for all $k \geq 0$

$$
\left\langle(A S-S A) e_{k}, e_{k+1}\right\rangle=\left\langle e_{k}, A^{*} v\right\rangle\left\langle u, e_{k+1}\right\rangle-\left\langle e_{k}, v\right\rangle\left\langle A u, e_{k+1}\right\rangle .
$$

Since $\left\langle(A S-S A) e_{k}, e_{k+1}\right\rangle=\left\langle A e_{k+1}, e_{k+1}\right\rangle-\left\langle A e_{k}, e_{k}\right\rangle$, it follows that

$$
\left(1+a_{k+1} \bar{b}_{k}\right)\left(\gamma_{k+1}-\gamma_{k}\right)=0 \text { for all } k \geq 0 .
$$

Then $\gamma_{k}=\gamma_{k+1}$ or $a_{k+1} \bar{b}_{k}=-1$ for all $k \geq 0$. If $\gamma_{k}=\gamma_{k+1}$ for all $k \geq 0$, then $A=\gamma I$ for some constant $\gamma$. Otherwise, $\gamma_{j+1} \neq \gamma_{j}$ for some $j$. If there are at most finitely many $j$ such that $\gamma_{j+1} \neq \gamma_{j}$, then $a_{j+1} \bar{b}_{j}=-1$ for such $j$. If there 
are infinitely many $j$ such that $\gamma_{j+1} \neq \gamma_{j}$, then $a_{j+1} \bar{b}_{j}=-1$ for such infinitely many $j$. But, since $\sum_{k=0}^{\infty}\left|a_{k}\right|^{2}<\infty$ and $\sum_{k=0}^{\infty}\left|b_{k}\right|^{2}<\infty, a_{j+1} \bar{b}_{j} \neq-1$ for sufficiently large $j$. So we have a contradiction. Thus there are at most finitely many $j$ satisfying $a_{j+1} \bar{b}_{j}=-1$. So we complete the proof.

The following example is an application of Theorem 2.12.

Example 2.13. Suppose that $u$ and $v$ are nonzero vectors in $\mathcal{H}$ and their expansions with respect to the orthonormal basis $\left\{e_{k}\right\}_{k=0}^{\infty}$ are $u=v=\sum_{k=0}^{\infty} \frac{1}{2^{k}} e_{n}$. Since $\frac{1}{2^{k+1}} \frac{1}{2^{k}} \neq-1$ for every nonnegative integer $k$, Theorem 2.12 implies that the commutant of $S+u \otimes v$ is $\gamma I$ for some constant $\gamma$.

The following proposition is very useful to decide whether the unilateral weighted shift in the commutant of $T=S+u \otimes v$ is hyponormal or not.

Proposition 2.14. Let $W \in \mathcal{L}(\mathcal{H})$ be the unilateral weighted shift with positive weight sequence $\left\{\gamma_{k}\right\}$ defined by $W e_{k}=\gamma_{k} e_{k+1}$ for $k=0,1,2, \ldots$ If $W \in\{T\}^{\prime}$ where $T=S+u \otimes v$, then for every nonnegative integer $k$, the following equation holds:

$$
\gamma_{k+1}\left(1+a_{k+1} \bar{b}_{k}\right)=\gamma_{k}\left(1+a_{k+2} \bar{b}_{k+1}\right) .
$$

Proof. Since the equation $W T=T W$ holds, we get that for every nonnegative integer $k$

$$
\left\langle(W S-S W) e_{k}, e_{k+2}\right\rangle=\left\langle e_{k}, W^{*} v\right\rangle\left\langle u, e_{k+2}\right\rangle-\left\langle e_{k}, v\right\rangle\left\langle W u, e_{k+2}\right\rangle .
$$

And we get that

$$
\begin{aligned}
\left\langle(W S-S W) e_{k}, e_{k+2}\right\rangle & =\left\langle W e_{k+1}, e_{k+2}\right\rangle-\left\langle S\left(\gamma_{k} e_{k+1}\right), e_{k+2}\right\rangle \\
& =\gamma_{k+1}-\gamma_{k} .
\end{aligned}
$$

Together,

Thus we have

$$
\gamma_{k+1}-\gamma_{k}=\gamma_{k} a_{k+2} \bar{b}_{k+1}-\gamma_{k+1} a_{k+1} \bar{b}_{k}
$$

for every nonnegative integer $k$.

Corollary 2.15. Let $W \in \mathcal{L}(\mathcal{H})$ be the unilateral weighted shift with positive weight sequence $\left\{\gamma_{k}\right\}$ defined by $W e_{k}=\gamma_{k} e_{k+1}$ for $k=0,1,2, \ldots$. Suppose that $T=S+u \otimes v$ satisfies $1+a_{k+1} \bar{b}_{k} \neq 0$ for every nonnegative integer $k$ and $W \in\{T\}^{\prime}$. Then $W$ is hyponormal if and only if $a_{k+2} \bar{b}_{k+1} \geq a_{k+1} \bar{b}_{k}$ for every nonnegative integer $k$.

Proof. If $W$ is hyponormal, it is known that the weight sequence $\left\{\gamma_{k}\right\}_{k=0}^{\infty}$ of $W$ is increasing. Hence from Proposition 2.14, we get that $\gamma_{k+1} \geq \gamma_{k}$ if and only if $1+a_{k+2} \bar{b}_{k+1} \geq 1+a_{k+1} \bar{b}_{k}$ for every nonnegative integer $k$. So we complete the proof.

Next examples show that the hyponormality of the unilateral weighted shift in the commutant of $T=S+u \otimes v$ depends on $u$ and $v$. 
Example 2.16. Suppose that $T=S+u \otimes v$ where $u=-\sum_{k=0}^{\infty} \frac{1}{k+1} e_{k}$ and $v=\sum_{k=0}^{\infty} \frac{1}{k+2} e_{k}$ and $W \in \mathcal{L}(\mathcal{H})$ is the unilateral weighted shift with positive weight sequence $\left\{\gamma_{k}\right\}$. If $W \in\{T\}^{\prime}$, then from Proposition 2.14 we get that

$$
\begin{aligned}
\frac{\gamma_{k+1}}{\gamma_{k}} & =\frac{1+a_{k+2} \bar{b}_{k+1}}{1+a_{k+1} \bar{b}_{k}} \\
& =\frac{1-\left(\frac{1}{k+3}\right)^{2}}{1-\left(\frac{1}{k+2}\right)^{2}}>1 .
\end{aligned}
$$

Thus the weight sequence $\left\{\gamma_{k}\right\}$ is increasing. Hence $W$ is hyponormal.

Example 2.17. Suppose that $T=S+u \otimes v$ where $u=\sum_{k=0}^{\infty} \frac{1}{2^{k}} e_{k}$ and $v=\sum_{k=0}^{\infty} \frac{1}{2^{k+1}} e_{k}$ and $W \in \mathcal{L}(\mathcal{H})$ is the unilateral weighted shift with positive weight sequence $\left\{\gamma_{k}\right\}$. If $W \in\{T\}^{\prime}$, then from Proposition 2.14 we obtain that

$$
\begin{aligned}
\frac{\gamma_{k+1}}{\gamma_{k}} & =\frac{1+a_{k+2} \bar{b}_{k+1}}{1+a_{k+1} \bar{b}_{k}} \\
& =\frac{1+\left(\frac{1}{2^{k+2}}\right)^{2}}{1+\left(\frac{1}{2^{k+1}}\right)^{2}}<1 .
\end{aligned}
$$

Thus the weight sequence $\left\{\gamma_{k}\right\}$ is decreasing. Hence $W$ is not hyponormal.

\section{References}

[1] A. Brown, On a class of operators, Proc. Amer. Math. Soc. 4 (1953), 723-728.

[2] A. Brown and C. Pearcy, Spectra of tensor products of operators, Proc. Amer. Math. Soc. 17 (1966), 162-166.

[3] G. Cassier and D. Timotin, Power boundedness and similarity to contractions for some perturbations of isometries, J. Math. Anal. Appl. 293 (2004), no. 1, 160-180.

[4] J. B. Conway, Subnormal operators, Pitman, London, 1981.

[5] _ A Course in Functional Analysis, Springer-Verlag, 1985.

[6] N. Dunford and J. Schwarz, Linear Operators III, John Wiley and Sons, 1971.

[7] P. R. Halmos, A Hilbert Space Problem Book, Springer-Verlag Berlin Heidelberg New York, 1980.

[8] E. Ionascu, Rank-one perturbations of diagonal operators, Integral Equations Operator Theory 39 (2001), no. 4, 421-440.

[9] I. Jung, E. Ko, and C. Pearcy, Aluthge transforms of operators, Integral Equations Operator Theory 37 (2000), no. 4, 437-448.

[10] C. Kitai, Invariant closed sets for linear operators, Ph. D. Thesis, Univ. of Toronto, 1982.

[11] M. Martin and M. Putinar, Lectures on Hyponormal Operators, Operator Theory: Advances and Applications, 39. Birkhäuser Verlag, Basel, 1989.

[12] Y. Nakamura, One-dimensional perturbations of the shift, Integral Equations Operator Theory 17 (1993), no. 3, 373-403.

[13] H. Radjavi and P. Rosenthal, Invariant Subspaces, Springer-Verlag, 1973.

[14] J. Stampfli, Perturbations of the shift, J. London Math. Soc. 40 (1965), 345-347.

[15] _ One-dimensional perturbations of operators, Pacific J. Math. 115 (1984), no. $2,481-491$. 
EungIL Ko

Department of Mathematics

Ewha Women's University

SeOul 120-750, Korea

E-mail address: eiko@ewha.ac.kr

Ji Eun LeE

Department of Mathematics

Kyung Hee University

SeOul 130-701, Korea

AND

Institute of Mathematical Sciences

EWHA WOMEN's UNIVERSITY

SEOUl 120-750, KoreA

E-mail address: jieun7@ewhain.net 